\title{
SPECTRAL MULTIPLIERS ON LIE GROUPS OF POLYNOMIAL GROWTH
}

\author{
G. ALEXOPOULOS
}

(Communicated by J. Marshall Ash)

\begin{abstract}
Let $L$ be a left invariant sub-Laplacian on a connected Lie group $G$ of polynomial volume growth, and let $\left\{E_{\lambda}, \lambda \geq 0\right\}$ be the spectral resolution of $L$ and $m$ a bounded Borel measurable function on $[0, \infty)$. In this article we give a sufficient condition on $m$ for the operator $m(L)=\int_{0}^{\infty} m(\lambda) d E_{\lambda}$ to extend to an operator bounded on $L^{p}(G), 1<p<\infty$, and also from $L^{1}(G)$ to weak- $L^{1}(G)$.
\end{abstract}

Let $G$ be a connected Lie group, and let us fix a left invariant Haar measure $d g$ on $G$. If $A$ is a Borel measurable subset of $G$, then we write $|A|=$ $d g$-measure $(A)$.

We assume that $G$ has polynomial volume growth, i.e., if $U$ is a compact neighborhood of the identity element $e$ of $G$, then there is a constant $c>0$ such that $\left|U^{n}\right| \leq c n^{c}, n \in \mathbb{N}$. Then $G$ becomes unimodular. Furthermore, there is an integer $D \geq 0$, such that (cf. Guivarc'h [7])

$$
\left|U^{n}\right| \sim n^{D} \quad(n \rightarrow \infty) .
$$

Notice that every connected nilpotent Lie group has polynomial volume growth.

Let $X_{1}, \ldots, X_{n}$ be left invariant vector fields on $G$ that satisfy Hörmander's condition, i.e., they generate together with their successive Lie brackets $\left[X_{i_{1}},\left[X_{i_{2}},\left[\ldots, X_{i_{k}}\right] \ldots\right]\right.$, at every point of $G$, the tangent space of $G$. To those vector fields is associated, in a canonical way, the control distance $d(\cdot, \cdot)$ (cf. [22, 23], which are our basic references for results concerning the Harmonic analysis on $G)$. This distance is left invariant and compatible with the topology on $G$. We put $|x|=d(e, x)$ and $B_{r}(x)=\{y \in G: d(x, y)<r\}, x \in G$, $r>0$. Then, we know that there is $d \in \mathbb{N}$, not depending on $x$ (cf. [15, 22]), such that

$$
\left|B_{r}(x)\right| \sim r^{d} \quad(r \rightarrow 0), \quad\left|B_{r}(x)\right| \sim r^{D} \quad(r \rightarrow \infty) .
$$

We consider the sub-Laplacian

$$
L=-\left(X_{1}^{2}+\cdots+X_{n}^{2}\right) .
$$

$L$ is a left-invariant hypoelliptic second-order differential operator (cf. [9]). It is also positive and selfadjoint (having as domain of definition the set $\{f \in$

Received by the editors April 24, 1992 and, in revised form, July 6, 1992.

1991 Mathematics Subject Classification. Primary 22E25, 22E30, 43A80.

Key words and phrases. Lie group, volume growth, multiplier, sub-Laplacian, wave equation. 
$\left.\left.L^{2}(G): L f \in L^{2}(G)\right\}\right)$. Hence it admits a spectral resolution which we denote by $\left\{E_{\lambda}, \lambda \geq 0\right\}$ (cf. [24]). If $m$ is a bounded Borel measurable function on $[0, \infty)$, then, using the spectral theorem, we can define the operator $m(L)=$ $\int_{0}^{\infty} m(\lambda) d E_{\lambda}$ which is bounded on $I^{2}(G)$.

We would like to know what conditions should be satisfied by $m$ so that $m(L)$ can be extended to an operator bounded on $L^{p}(G), p \in(1, \infty)$. In particular, we would like to generalize the classical, in the case of $\mathbb{R}^{n}$, theorems of Hörmander [8] and Mikhlin [14].

This question has already been studied in the particular case where $G$ is a stratified nilpotent Lie group (then we have $d=D$ and $L$ is invariant with respect to the dilation structure of $G$ ); see, for example, the result of Hulanicki and Stein [6, pp. 208-215], De Michele and Mauceri [5], Hulanicki and Jenkins [10], Mauceri and Meda [12], and Christ [3] (see also the remarks at the end of this article).

This type of question has also been studied, with different methods, in the case of symmetric spaces (cf. Clerc and Stein [4], Stanton and Tomas [18], Anker and Lohoué [2], Taylor [21], and Anker [1]).

Finally Stein [20] has proved a very general result (which applies, for example, when $m$ is of the Laplace tranform type) but with much more restrictive conditions.

The goal of this article is to prove the following result:

Theorem. Let $m(L), d, D$ be as above, and put $\alpha=[d / 2]+1, A=[D / 2]+1$, and $k \wedge \alpha=\min (k, \alpha)$. If the function $m$ is such that

$$
\begin{aligned}
& \text { if } d \geq D \quad \begin{cases}\sup _{0<\lambda \leq 1} \lambda^{k}\left|m^{(k)}(\lambda)\right|<\infty, & 0 \leq k \leq A, \\
\sup _{\lambda>1} \lambda^{k}\left|m^{(k)}(\lambda)\right|<\infty, & 0 \leq k \leq \alpha ;\end{cases} \\
& \text { if } d<D \quad \begin{cases}\sup _{0<\lambda \leq 1} \lambda^{k}\left|m^{(k)}(\lambda)\right|<\infty, & 0 \leq k \leq A, \\
\sup _{\lambda>1} \lambda^{k \wedge \alpha}\left|m^{(k)}(\lambda)\right|<\infty, & 0 \leq k \leq A,\end{cases}
\end{aligned}
$$

then $m(L)$ is bounded on $L^{p}(G), 1<p<\infty$, and also from $L^{1}(G)$ to weak$L^{1}(G)$.

The proof of the above theorem contains the following corollary, which generalizes a result of Hulanicki and Jenkins [10].

Corollary. Let $m(L), D$ and $A$ be as in the above theorem, and assume that the support of $m$ is a compact subset of $(0, \infty)$ and that $m \in C^{A}(\mathbb{R})$. Then $m(L)$ is given by convolution with a kernel that belongs to $L^{1}(G)$.

Our proof is similar to the proof of Hulanicki and Stein [6] and Christ [3], in the sense that it relies heavily on good estimates of the heat kernel. But in our case the situation is more compliated because of the absense of any "convenient" dilation structure. To overcome this difficulty, we use an idea of Taylor [21], which, roughly speaking, consists of writing $m(L)=f\left(L^{1 / 2}\right)$ with $f$ an even function, and then using the formula $f\left(L^{1 / 2}\right)=(2 \pi)^{-1 / 2} \int_{-\infty}^{\infty} \hat{f}(t) \cos t L^{1 / 2} d t$ to take advantage of the $L^{2}$-boundedness and the finite propagation speed of the operator $\cos t L^{1 / 2}$. This last fact has been proved, for subelliptic operators, by Melrose [13]. 
Proof of the theorem. Before we start the proof of the theorem we shall state the following

Lemma. Assume that the function $f(x) \in C(\mathbb{R})$ has compact support and that it possesses $n$ continuous derivatives $f^{\prime}(x), f^{\prime \prime}(x), \ldots, f^{(n)}(x)$, and let $M_{\alpha}=$ $\sup \left\{\left|f^{(n)}(x+t)-f^{(n)}(x)\right| / t^{\alpha}, t>0, x \in \mathbb{R}\right\}, 0<\alpha \leq 1$. Then for every $\lambda>0$ there is an even bounded integrable function $\psi_{\lambda}(x) \in C(\mathbb{R})$ such that

$$
\operatorname{supp}\left(\hat{\psi}_{\lambda}\right) \subseteq[-\lambda, \lambda] \quad \text { and } \quad\left|f(x)-f * \psi_{\lambda}(x)\right| \leq c \frac{M_{\alpha}}{\lambda^{n+\alpha}}, \quad x \in \mathbb{R}
$$

( $c$ is a constant that depends only on $n$ ).

The proof of the above lemma is exactly the same as in the case when $f(x)$ is periodic (cf. $[11$, p. $57 ; 16$, p. 88]). Let us only say that it is done by induction on $n$ and that, instead of using Jackson's kernel $U_{n}(x)=k_{n}[\sin (n x / 2) / \sin (x / 2)]^{4}$, $n \in \mathbb{N} \quad\left(k_{n}\right.$ is a constant making the integral $\left.\int_{0}^{2 \pi} U_{n}(x) d x=1\right)$, we use its analogue for $\mathbb{R}, H_{\lambda}(x)=k_{\lambda}[\sin (\lambda x / 2) /(x / 2)]^{4}, \lambda>0$ (again $k_{\lambda}$ is defined by the condition $\left.\int_{-\infty}^{\infty} H_{\lambda}(x) d x=1\right)$.

We denote by $p_{t}(x, y)$ the heat kernel, i.e., the kernel of the semigroup $e^{-t L}, t>0$. Since $L$ is symmetric and left invariant, we have that $p_{t}(x, y)=$ $p_{t}(y, x)$ and that $p_{t}(x, y)=p_{t}\left(e, x^{-1} y\right), x, y \in G, t>0$. We put $p_{t}(x)=$ $p_{t}(e, x)$. [22]):

We shall need the following estimates of the kernel $p_{t}(x)$ (cf. Varopoulos

$$
\begin{array}{ll}
p_{t}(x) \leq C t^{-d / 2} \exp \left(-c|x|^{2} / t\right), & 0<t \leq 1, \\
p_{t}(x) \leq C t^{-D / 2} \exp \left(-c|x|^{2} / t\right), & t>1,
\end{array}
$$

and of its derivatives $X_{i} p_{t}, 1 \leq i \leq n$ (cf. Salof-Coste [17]):

$$
\begin{array}{ll}
\left|X_{i} p_{t}(x)\right| \leq C t^{-(d+1) / 2} \exp \left(-c|x|^{2} / t\right), & 0<t \leq 1, \\
\left|X_{i} p_{t}(x)\right| \leq C t^{-(D+1) / 2} \exp \left(-c|x|^{2} / t\right), & t>1
\end{array}
$$

$(c, C>0$ are constants not depending on $t$ and $x$ ).

We shall also use the following property of the Schwartz kernel $G_{t}(x, y)$ of the operator $\cos t L^{1 / 2}$ (cf. Melrose [13]):

$$
\operatorname{supp}\left(G_{t}\right) \subseteq\left\{(x, y) \in G \times G:\left|x^{-1} y\right| \leq|t|\right\} .
$$

Now we are ready to start the proof of the theorem. First of all, following [3], we observe that it follows from (4) that $\lim _{t \rightarrow \infty}\left\|p_{t} * f\right\|_{2}=0$, i.e., that $\lim _{t \rightarrow \infty}\left\|e^{-t L} f\right\|_{2}=0$. This in turn implies that $\lim _{\varepsilon \rightarrow 0}\left\|\int_{0}^{\varepsilon} d E_{\lambda}(f)\right\|_{2}=0$. So the point $\lambda=0$ may be neglected in the spectral resolution of $L$, and therefore we can consider that the function $m(\lambda)$ is defined on $(0, \infty)$. that

Next, following Hörmander [8], we consider a function $\phi \in C_{0}^{\infty}\left(\mathbb{R}^{+}\right)$such

$$
\operatorname{supp} \phi \subseteq\left(\frac{1}{2}, 2\right), \quad \sum_{j \in \mathbb{Z}} \phi\left(2^{j} t\right)=1, \quad t>0 .
$$

We put, for all $j \in \mathbb{Z}$,

$$
m_{j}(\lambda)=m(\lambda) \phi\left(2^{-j} \lambda\right), \quad f_{j}(\lambda)=m_{j}(\lambda) \exp \left(2^{-j} \lambda\right), \quad h_{j}(\lambda)=f_{j}\left(\lambda^{2}\right) .
$$


Observe that it follows from (2) that

$$
\begin{array}{ll}
\sup _{\lambda \in \mathbb{R}}\left|h_{j}^{(A)}(\lambda)\right| \leq c 2^{-A j / 2} & \text { if } j<0 ; \\
\sup _{\lambda \in \mathbb{R}}\left|h_{j}^{(\alpha)}(\lambda)\right| \leq c 2^{-\alpha j / 2} & \text { if } j \geq 0 ; \\
\sup _{\lambda \in \mathbb{R}}\left|h_{j}^{(A)}(\lambda)\right| \leq c 2^{-\alpha j / 2} & \text { if } j \geq 0, D>d .
\end{array}
$$

Making use of the lemma we consider, for all $j, p \in \mathbb{Z}$, an even function $\psi_{j, p}$ such that

$$
\operatorname{supp} \hat{\psi}_{j, p} \subseteq\left[-2^{p / 2-2}, 2^{p / 2-2}\right]
$$

and such that for some constant $c>0$ we have

$$
\begin{array}{ll}
\sup _{t \in \mathbb{R}}\left|h_{j}(t)-h_{j} * \psi_{j, p}(t)\right| \leq c 2^{-A j / 2} 2^{-A p / 2} & \text { if } j<0 ; \\
\sup _{t \in \mathbb{R}}\left|h_{j}(t)-h_{j} * \psi_{j, p}(t)\right| \leq c 2^{-\alpha j / 2} 2^{-\alpha p / 2} & \text { if } j \geq 0, d \geq D ; \\
\sup _{t \in \mathbb{R}}\left|h_{j}(t)-h_{j} * \psi_{j, p}(t)\right| \leq c 2^{-\alpha j / 2} 2^{-\alpha p / 2} & \text { if } j \geq 0, p \leq 0, d<D ; \\
\sup _{t \in \mathbb{R}}\left|h_{j}(t)-h_{j} * \psi_{j, p}(t)\right| \leq c 2^{-\alpha j / 2} 2^{-A p / 2} & \text { if } j \geq 0, p>0, d<D .
\end{array}
$$

Let $K$ and $K_{j}$ the Schwartz kernels of the operators $m(L)$ and $m_{j}(L)$ respectively. Since the operators $m(L)$ and $m_{j}(L)$ are left invariant (they are also symmetric), these are convolution kernels, i.e., $\left(m(L) f=f * K, m_{j}(L) f=\right.$ $f * K_{j}$ ). Then we have

$$
m_{j}(L)=f_{j}(L) e^{-2^{-j} L}, \quad f_{j}(L)=h_{j}\left(L^{1 / 2}\right),
$$

and therefore

$$
K_{j}(x)=f_{j}(L) p_{2^{-j}}(x)=(2 \pi)^{-1 / 2} \int_{-\infty}^{+\infty} \hat{h}_{j}(t) \cos t L^{1 / 2} p_{2^{-j}}(x) d t
$$

We want to estimate the quantity

$$
I(t, j)=\int_{\{|x| \geq 2 t\}}\left|K_{j}(x)-K_{j}\left(y^{-1} x\right)\right| d x, \quad t>0,|y| \leq t .
$$

To this end we put $A_{p}=\left\{x \in G: 2^{p / 2} \leq|x|<2^{p / 2+1 / 2}\right\}$ and consider an integer $\tau \in \mathbb{Z}$ such that $2^{\tau / 2} \leq t<2^{\tau / 2+1 / 2}$. Then we have

$$
\begin{aligned}
I(t, j) \leq & \sum_{p \geq \tau+2}\left|A_{p}\right|^{1 / 2}\left\|K_{j}(\cdot)-K_{j}\left(y^{-1} \cdot\right)\right\|_{L^{2}\left(A_{p}\right)} \quad \text { if }-j \leq \tau, \\
I(t, j) \leq & \left|B_{2-j / 2}\right|^{1 / 2}\left\|K_{j}(\cdot)-K_{j}\left(y^{-1} \cdot\right)\right\|_{2} \\
& +\sum_{p \geq-j}\left|A_{p}\right|^{1 / 2}\left\|K_{j}(\cdot)-K_{j}\left(y^{-1} \cdot\right)\right\|_{L^{2}\left(A_{p}\right)} \quad \text { if }-j>\tau .
\end{aligned}
$$

Now, on the one hand we have

$$
\left\|K_{j}(\cdot)-K_{j}\left(y^{-1} \cdot\right)\right\|_{2} \leq c\left\|p_{2-j}(\cdot)-p_{2-j}\left(y^{-1} \cdot\right)\right\|_{2}
$$


On the other hand, following Taylor [21], we observe that it follows from (6) and (8) that

$$
\begin{aligned}
& K_{j}(x)-\left.K_{j}\left(y^{-1} x\right)\right|_{A_{p}} \\
& =(2 \pi)^{-1 / 2} \int_{-\infty}^{+\infty} \hat{h}_{j}(s) \cos s L^{1 / 2}\left\{\left[p_{2^{-j}}(\cdot)-p_{2^{-j}}\left(y^{-1} \cdot\right)\right] 1_{\left\{|x| \leq 2^{(p-1) / 2}\right\}}\right. \\
& \left.+\left[p_{2^{-j}}(\cdot)-p_{2^{-j}}\left(y^{-1} \cdot\right)\right] 1_{\left\{|x|>2^{(p-1) / 2}\right\}}\right\}(x) d s \\
& =(2 \pi)^{-1 / 2} \int_{-\infty}^{+\infty}\left[\hat{h}_{j}(s)-\hat{h}_{j}(s) \hat{\psi}_{j, p}(s)\right] \\
& \times \cos s L^{1 / 2}\left\{\left[p_{2^{-j}}(\cdot)-p_{2^{-j}}\left(y^{-1} \cdot\right)\right] \mathbf{1}_{\left\{|x| \leq 2^{(p-1) / 2}\right\}}\right\}(x) d s \\
& +(2 \pi)^{-1 / 2} \int_{-\infty}^{+\infty} \hat{h}_{j}(s) \cos s L^{1 / 2}\left\{\left[p_{2^{-j}}(\cdot)-p_{2^{-j}}\left(y^{-1} \cdot\right)\right] 1_{\left\{|x|>2^{(p-1) / 2}\right\}}\right\}(x) d s,
\end{aligned}
$$

and therefore

(11)

$$
\begin{aligned}
\left\|K_{j}(\cdot)-K_{j}\left(y^{-1} \cdot\right)\right\|_{L^{2}\left(A_{p}\right)} \leq & \left\|\left[p_{2^{-j}}(\cdot)-p_{2^{-j}}\left(y^{-1} \cdot\right)\right] 1_{\left\{|x| \leq 2^{(p-1) / 2}\right\}}\right\|_{2} \\
& \times \sup _{t \in \mathbb{R}}\left|h_{j}(t)-h_{j} * \psi_{j, p}(t)\right| \\
& +\sup _{t \in \mathbb{R}}\left|h_{j}(t)\right|\left\|\left[p_{2^{-j}}(\cdot)-p_{2^{-j}}\left(y^{-1} \cdot\right)\right] 1_{\left\{|x|>2^{(p-1) / 2}\right\}}\right\|_{2} .
\end{aligned}
$$

Now, the estimation of $I(t, j)$ is just a matter of putting everything together and calculating. More precisely, the estimate of the different quantities appearing in (9) is done as follows ( $c, C$ denote universal constants, $c, C>0)$ :

(a) $\left|A_{p}\right|$ and $\left|B_{2^{j / 2}}\right|$ are estimated by making use of (1).

(b) The estimation of $\left\|K_{j}(\cdot)-K_{j}\left(y^{-1} \cdot\right)\right\|_{2}$ is reduced, in view of (10), to the estimation of $\left\|p_{2^{-j}}(\cdot)-p_{2^{-j}}\left(y^{-1} \cdot\right)\right\|_{2}$, which in turn is estimated by making use of (4); for example, if $j \geq 0$ then

$$
\left\|p_{2^{-j}}(\cdot)-p_{2^{-j}}\left(y^{-1} \cdot\right)\right\|_{2} \leq c|y| 2^{j / 2} 2^{d j / 4} .
$$

(c) To estimate $\left\|K_{j}(\cdot)-K_{j}\left(y^{-1} \cdot\right)\right\|_{L^{2}\left(A_{p}\right)}$ we make use of (11) where:

(i) the term $\sup _{t \in \mathbb{R}}\left|h_{j}(t)-h_{j} * \psi_{j, p}(t)\right|$ is estimated by making use of (7);

(ii) the term $\left\|\left[p_{2-j}(\cdot)-p_{2-j}\left(y^{-1} \cdot\right)\right] 1_{\{|x|>2(p-1) / 2}\right\|_{2}$ is estimated by making use of (3) and (4); for example, if $-j<\tau$ and $j \geq 0$, then

$$
\left\|\left[p_{2-j}(\cdot)-p_{2-j}\left(y^{-1} \cdot\right)\right] 1_{\left\{|x|>2^{(p-1) / 2}\right\}}\right\|_{2} \leq C 2^{d j / 4} \exp \left(-c 2^{j+p}\right),
$$

and if $-j \geq \tau$ and $j \geq 0$, then

$$
\left\|\left[p_{2^{-j}}(\cdot)-p_{2^{-j}}\left(y^{-1} \cdot\right)\right] 1_{\left\{|x|>2^{(p-1) / 2}\right\}}\right\|_{2} \leq C|y| 2^{j / 2} 2^{d j / 4} \exp \left(-c 2^{j+p}\right),
$$

and finally

(iii) the term $\left\|\left[p_{2^{-j}}(\cdot)-p_{2^{-j}}\left(y^{-1} \cdot\right)\right] \mathbf{1}_{\left\{|x| \leq 2^{(p-1) / 2}\right\}}\right\|_{2}$ is estimated by simply writing

$$
\left\|\left[p_{2-j}(\cdot)-p_{2^{-j}}\left(y^{-1} \cdot\right)\right] 1_{\left\{|x| \leq 2^{(p-1) / 2}\right\}}\right\|_{2} \leq\left\|p_{2-j}(\cdot)-p_{2-j}\left(y^{-1} \cdot\right)\right\|_{2}
$$

and then proceeding as in the cases (a) and (c)(ii) above, i.e., by distinguishing the cases $-j<\tau$ and $-j \geq \tau$ and then using (3) and (4). 
By substituting into (9) and taking the sums, we conclude that there is a constant $c>0$ such that

$$
\begin{aligned}
& I(t, j) \leq c\left(t 2^{j / 2}\right)^{-1 / 2} \quad \text { if } t 2^{j / 2} \geq 1, \\
& I(t, j) \leq c t 2^{j / 2} \quad \text { if } t 2^{j / 2}<1 .
\end{aligned}
$$

This in turn implies that there is a constant $c>0$ such that for all $t>0$ and $|y| \leq t$, we have

$$
\int_{|x| \geq 2 t}\left|K(x)-K\left(y^{-1} x\right)\right| d x \leq \sum_{j \in \mathbb{Z}} I(t, j) \leq c .
$$

Finally, we conclude by making use of a classical result of Hörmander [8] which affirms that (13), together with the fact that the operator $m(L)$ is bounded and symmetric on $L^{2}(G)$, imply that $m(L)$ is bounded on $L^{p}(G), 1<p<\infty$, and also from $L^{1}(G)$ to weak- $L^{1}(G)$, and the theorem follows.

Final remarks. We stated condition (2) of the theorem in terms of an integral number of derivatives, because in this way it is better illustrated what happens when $D>d$. In terms of Hölder norms, condition (2) could be replaced, in the case $D \leq d$ for example, by the condition

$$
\begin{gathered}
\sup _{0<t \leq 1}\|\varphi(\cdot) m(t \cdot)\|_{C^{D / 2+\varepsilon}(\mathbf{R})}<\infty \\
\sup _{t \geq 1}\|\varphi(\cdot) m(t \cdot)\|_{C^{d / 2+\varepsilon}(\mathbf{R})}<\infty
\end{gathered}
$$

where $\varepsilon$ is any positive number $\varepsilon>0$ and $\varphi$ is an auxilliary function, not identically zero, $\varphi \in C_{0}^{\infty}\left(\mathbb{R}^{+}\right)$. The proof is the same. The only change is that the exponent $-\frac{1}{2}$ in (12) will be replaced by $-\varepsilon$.

In terms of Sobolev norms, however, the best we can do is to ask for $m(\lambda)$ to satisfy the condition (always in the case $D \leq d$ )

$$
\begin{gathered}
\sup _{0<t \leq 1}\|\varphi(\cdot) m(t \cdot)\|_{L_{D / 2+1 / 2+\varepsilon}^{2}(\mathbf{R})}<\infty, \\
\sup _{t \geq 1}\|\varphi(\cdot) m(t \cdot)\|_{L_{d / 2+1 / 2+\varepsilon}^{2}(\mathbf{R})}<\infty
\end{gathered}
$$

where $L_{\alpha}^{2}$ denotes the $L^{2}$ Sobolev space of order $\alpha$ and $\varphi$ and $\varepsilon$ are as in (14). Of course, the proof in this case can be reduced to the proof under condition (14) by using the fact that $L_{\alpha}^{2}(\mathbb{R}) \subseteq C^{k}(\mathbb{R})$, if $\alpha>k+\frac{1}{2}$. However, under condition (15), the proof of the theorem is much simpler, since the lemma is no longer required. We can simply substitute the term $\sup _{t \in \mathbb{R}}\left|h_{j}(t)-h_{j} * \psi_{j, p}(t)\right|$ in (11) by the term $\int_{|t| \geq 2 p / 2-2}\left|\hat{h}_{j}(t)\right| d t$, which in turn can be estimated in terms of the Sobolev norm of the function $h_{j}(t)$.

So our result, although more general, is weaker than the result of Christ [3] and Mauceri and Meda [12] who, in the particular case where $G$ is a stratified nilpotent Lie group (then we have $d=D$ ) and $L$ is dilation invariant, prove the theorem under the weaker condition $\sup _{t>0}\|\varphi(\cdot) m(t \cdot)\|_{L_{D / 2+\varepsilon}^{2}(\mathbf{R})}<\infty$ (again $\varphi, \varepsilon$, and $\|\cdot\|_{L_{\alpha}^{2}(\mathbb{R})}$ are as in (14)). 


\section{ACKNOWLEDGMENT}

I thank M. Christ for several helpful discussions. I also thank N. Th. Varopoulos and N. Lohoué for their constant help and encouragement.

\section{BibLIOGRAPHY}

1. J.-Ph. Anker, $L^{p}$ Fourier multipliers on Riemannian symmetric spaces of the noncompact type, Ann. of Math. (2) 132 (1990), 597-628.

2. J.-Ph. Anker and N. Lohoué, Multiplicateurs sur certains espaces symétriques, Amer. J. Math. 108 (1986), 1303-1354.

3. M. Christ, $L^{p}$ bounds for spectral multipliers on nilpotent groups, Trans. Amer. Math. Soc. 328 (1991), 73-81.

4. J.-L. Clerc and E. M. Stein, $L^{p}$-multipliers for noncompact symmetric spaces, Proc. Nat. Acad. Sci. U.S.A. 71 (1974), 3911-3912.

5. L. De Michele and G. Mauceri, $H^{p}$ multipliers on stratified groups, Ann. Mat. Pura Appl. 148 (1987), 353-366.

6. G. B. Folland and E. Stein, Hardy spaces on homogeneous groups, Princeton Univ. Press, NJ, 1982.

7. Y. Guivarc'h, Croissance polynômiale et périodes de fonctions harmoniques, Bull. Sci. Math. France 101 (1973), 149-152.

8. L. Hörmander, Estimates for translation for translation invariant operators in $L^{p}$ spaces, Acta Math. 104 (1960), 93-139.

9. __ Hypoelliptic second order differential operators, Acta Math. 119 (1967), 283-323.

10. A. Hulanicki and J. W. Jenkins, Almost everywhere summability on nilmanifolds, Trans. Amer. Math. Soc. 278 (1983), 703-715.

11. G. G. Lorenz, Approximation of functions, Holt, Reinehart, and Wilson, New York, 1966.

12. G. Mauceri and S. Meda, Vector-valued multipliers on stratified groups, Rev. Mat. Iberoamericana 6 (1990), 141-154.

13. R. Melrose, Propagation for the wave group of a positive subelliptic second order differential operator, Tanigushi Sympos. HERT (Katata, 1984) (S. Mizohata, ed.), Academic Press, New York, 1986, pp. 181-192.

14. S. Mikhlin, Multidimensional singular integral equations, Pergamon Press, New York, 1965.

15. A. Nagel, E. Stein, and M. Wainger, Balls and metrics defined by vector fields, Acta Math. 155 (1985), 103-147.

16. I. P. Natanson, Constructive function theory, vol. 1: uniform approximation, Ungar, New York, 1964.

17. L. Saloff-Coste, Analyse sur les groupes de Lie à croissance polynômiale, Ark. Mat. 28 (1990), 315-331.

18. R. J. Stanton and P. A. Tomas, Expansions for spherical functions on noncompact symmeiric spaces, Acta Math. 140 (1978), 251-276.

19. E. Stein, Singular integrals and differentiability properties of functions, Princeton Univ. Press, Princeton, NJ, 1970.

20. E. Stein, Topics in harmonic analysis, Princeton Univ. Press, Princeton, NJ, 1970.

21. M. E. Taylor, $L^{p}$ estimates on functions on the Laplace operator, Duke Math. J. 58 (1989), 773-793.

22. N. Th. Varopoulos, Analysis on Lie groups, J. Funct. Anal. 76 (1988), 346-410.

23. N. Th. Varopoulos, L. Saloff-Coste, and T. Coulhon, Analysis and geometry on groups, Cambridge Tracts in Math., Cambridge Univ. Press, New York, 1992.

24. K. Yosida, Functional analysis, Springer-Verlag, New York, 1978.

Universite de Paris-Sud, Mathematiques, Bât. 425, 91405 Orsay Cedex, France 